Methods The study includes data from the Danish Occupational Medicine Cohort. 17,900 patients aged 16-67 were included. All patients were referred to a department of occupational medicine in Denmark from 2000-2013 due to suspected work-related mental health problems. Register data was extracted 5 years prior to study inclusion and until 5 years after study inclusion. Information on 13 potential risk factors for negative labor market attachment was included. The primary outcome of this study was positive work status, defined as being at work or at education, maternity or parental leave. Results Preliminary results showed that approximately $85 \%$ of all patients from 2-5 years prior to study inclusion had positive work status. The proportion declined to $30 \%$ at the year of study inclusion and increased to approximately 50\% of the patients 1 year after inclusion after which practically no further increase was seen. Within the subgroups of patients diagnosed with depression or PTSD only $40 \%$ and $30 \%$ had positive work status 5 years after study inclusion respectively. Besides, preliminary results indicated that diagnosis, sick leave status at inclusion time and previous work participation, were strong independent prognostic markers of work participation at 5-years of follow up.

Conclusion We found a profoundly reduced long-term employment status among patients referred to departments of occupational medicine in Denmark due to suspected work-related mental health problems, particularly among patients diagnosed with PTSD or depression.

\section{0-271 WORK FUNCTIONING AMONG YOUNG ADULTS: THE ROLE OF MENTAL HEALTH PROBLEMS FROM CHILDHOOD TO YOUNG ADULTHOOD}

'Samira de Groot, Karin Veldman, Benjamin C Amick, Ute Bültmann. 'University of Groningen and University Medical Centre Groningen, Netherlands

\subsection{6/OEM-2021-EPI.109}

Introduction Mental health problems during childhood and adolescence are negatively associated with employment status (having a paid job or not) in young adulthood. Yet, little is known about how young adults function at work, i.e., do they experience difficulties in meeting job demands given their physical or mental health state.

Objective This longitudinal study aims to examine whether the history of mental health problems from childhood to young adulthood is associated with work functioning in young adulthood.

Methods Data were used from 1,004 young adults in the TRacking Adolescents' Individual Lives Survey (TRAILS), a Dutch prospective cohort study with 18-year follow-up and seven measurement waves. Mental health problem trajectories including $11,13,16,19,22$ and 26 age points were identified using growth mixture models. Work functioning was assessed at age 29 with the Work Role Functioning Questionnaire, with scores ranging from 0 to 100 . Regression analyses were conducted to examine the associations between mental health problems trajectories and work functioning.

Results Young adults with high-stable trajectories of internalising or externalising problems reported lower work functioning (respectively 74.6 and 76.2) compared to participants with low-stable trajectories (respectively 83.3 and 83.2). These scores correspond with reduced work functioning for more than one working day per week in a fulltime job. Young adults with moderate-stable trajectories of externalising problems reported lower work functioning compared to participants with low-stable trajectories.

Conclusion Persistent high-level mental health problems during childhood, adolescence and young adulthood are associated with lower work functioning in young adulthood compared to those with low-level mental health problems. As information on work functioning provides insight into the difficulties young workers experience in meeting their job demands, it can be used as a starting point for a conversation between occupational physicians and young workers to address these difficulties and to increase work functioning.

\section{0-340 JOB STRAIN, LOW SOCIAL SUPPORT FROM SUPERVISORS AND MATERIAL HANDLING AMONG BRAZILIAN WORKERS: A STUDY ON ETHNICITY AND WORK}

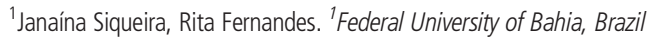

\subsection{6/OEM-2021-EPI.110}

Introduction The contribution of race/color to outcomes with a potential effect on worker's health needs further exploration on epidemiological occupational surveys.

Objectives The study proposed to analyze the association between job strain, social support from supervisor and material handling at work according to the self-reported race/color. Methods This cross-sectional study involved 1032 workers from urban cleaning service and footwear manufacturers located in the state of Bahia, Brazil. The interview included the Job Content Questionnaire to measure psychosocial exposure and questions about lifting, pushing and pulling to investigate material handling in the workday. Cox Regression provided brute and adjusted prevalence ratios (PRadj) by sex, age and education.

Results Black workers presented higher proportion of lower education and were more represented in operational positions, followed by brown, both compared with white workers. There was a greater exposure of blacks to job strain (PRadj $=1.6$ ), a combination of high psychological demand and low job control, and low social support from supervisors (PRadj $=1.3$ ). Concomitantly, blacks were more subjected to activities with pushing, pulling and lifting materials, presenting PRadj at least $50 \%$ higher compared to white workers. Brown were slightly more exposed to job strain (PRadj $=1.1$ ), and to the low social support from supervisors (PRadj $=1.1$ ), and were also more subjected to lifting $($ PRadj $=1.2)$, pushing $($ PRadj $=$ 1.3) and pulling $(\mathrm{PRadj}=1.2)$ materials compared to whites. The complete results of this epidemiological research are available in the brazilian periodic Cience \& Coletive Health (online first).

Conclusion The study evidenced an overlap of disadvantageous exposures among black and brown brazilian workers, accentuated among blacks, that can indicate the repercussions of structural racism in the world of work and should be the target of interventions focusing on improving working conditions and racial equity. 